\title{
Spin Correlated-Plasmons at Room Temperature Driven by Electronic Correlations in Lead-Free 2D Hybrid Organic-Inorganic Perovskites
}

Muhammad Avicenna Naradipa ${ }^{1,2}$, Aozhen Xie $e^{3,4,5}$ Arramel $^{1}$, Xinmao Yin ${ }^{1,2}$, Chi Sin Tang $^{1,2,6}$, Muhammad Fauzi Sahdan ${ }^{1}$, Teguh Citra Asmara ${ }^{7}$, Cuong Dang ${ }^{3,4,5}$, Muhammad Danang Birowosuto ${ }^{3, *}$, Andrew Thye Shen We $e^{1,2,8}$, and Andrivo Rusydi, 1,2,6,9,*

${ }^{1}$ Department of Physics, National University of Singapore, 2 Science Drive 3, 117551

Singapore

${ }^{2}$ Singapore Synchrotron Light Source, National University of Singapore, 5 Research Link, Singapore 1176033

${ }^{3}$ CINTRA UMI CNRS/NTU/THALES 3288, Research Techno Plaza, 50 Nanyang Drive, Border X Block, Level 6, Singapore 637553, Singapore

${ }^{4}$ School of Electrical and Electronics Engineering, Nanyang Technological University, 50 Nanyang Avenue, Singapore 639798, Singapore

${ }^{5}$ Energy Research Institute @NTU (ERI@N), Research Techno Plaza, X-Frontier Block, Level 5, 50 Nanyang Drive, Singapore 637553, Singapore

${ }^{6}$ NUS Graduate School for Integrative Sciences and Engineering (NGS), National University of Singapore, Singapore 117456

${ }^{7}$ Photon Science Division, Swiss Light Source, Paul Scherrer Institute, Forschungsstrasse 111, CH-5232, Villigen PSI, Switzerland

${ }^{8}$ Centre for Advanced 2D Materials, National University of Singapore, Block S14, Level 6, 6 Science Drive 2, Singapore 117546

${ }^{9}$ NUSNNI-NanoCore, National University of Singapore, Singapore 117411

*Corresponding author: mbirowosuto@ntu.edu.sg and phyandri@nus.edu.sg andrivo.rusydi@nus.edu.sg 


\section{$\underline{\text { Methods }}$}

\section{(MA) $)_{2} \mathrm{CuCl}_{4}$ Single Crystal Synthesis}

Our sample fabrication process for (MA) $\mathrm{CuCl}_{4}$ is a slow evaporation method of precursors using methylammonium chloride ( $\mathrm{MACl}, \geq 98 \%$ ) and copper chloride $\left(\mathrm{CuCl}_{2}, \geq 99 \%\right)$. They were purchased from Sigma Aldrich and used without further purification. Furthermore, the precursors were dissolved in water with a 2:1 molar ratio to prepare the $1 \mathrm{M}(\mathrm{MA})_{2} \mathrm{CuCl}_{4}$ precursor solution ${ }^{1}$. We used $1 \mathrm{~mL}$ precursor solutions in a $25-\mathrm{mL}$ vial that were kept undisturbed at room temperature to allow slow evaporation of water. Typically, within one week, a film of green-orange (MA) ${ }_{2} \mathrm{CuCl}_{4}$ can be obtained. Although (MA) ${ }_{2} \mathrm{CuCl}_{4}$ will degrade after a few weeks of exposure to humidity in the air, the samples are relatively stable for one week with 6-8 hours of room temperature measurement. As a comparison, we also fabricated (MA) ${ }_{2} \mathrm{CdCl}_{4}$ and (MA) $\mathrm{ZnCl}_{4}$ using similar techniques (for (MA) $\mathrm{ZnCl}_{4}$, an additional $40^{\circ} \mathrm{C}$ heating is done to evaporate the water from the solution).

\section{Atomic Force Microscopy (AFM)}

The sample morphology was characterized using a commercial atomic force microscope (AFM, BRUKER Dimension FastScan). A standard cantilever with spring constant of $40 \mathrm{~N} / \mathrm{m}$ and tip curvature $<10 \mathrm{~nm}$ was used as the probe.

\section{X-Ray Diffraction (XRD)}

High resolution X-ray diffractometry characterizations for the samples were performed at the Xray Demonstration and Development (XDD) beamline at the Singapore Synchrotron Light Source (SSLS). All diffraction measurements were carried out at room temperature. X-ray diffractograms data acquisitions were done in two-axis mode with a step size of $0.02^{\circ}$ over a Bragg angle range between $10-80^{\circ}$.

\section{Scanning Electron Microscopy (SEM)}

SEM micrographs were taken under $6 \mathrm{kV}$ accelerating voltage in secondary electron mode using JEOL JSM-5600LV system.

\section{Photoluminescence and Absorption Spectroscopies}

Room temperature Photoluminescence (PL) measurements were performed using free space excitation and collection through a visible-near infrared microscope objective (Nikon 20x, NA = 
0.40). The samples were excited with a ps-pulsed ytterbium fiber laser emitting at high harmonic generation of $355 \pm 1 \mathrm{~nm}$ wavelength with $20 \mathrm{MHz}$ repetition rate, focused to a spot size of less than $1 \mu \mathrm{m}$. Photoluminescence was detected using a Peltier-cooled photomultiplier tube (Hamamatsu H7422 series) coupled to a grating spectrometer (Edinburgh Instruments F900 and Bentham TMS300). Time-resolved decay traces were acquired by a time-correlated single photon counting acquisition module (Edinburgh Instruments, TCC900) at selected emission wavelength of $425 \pm 5 \mathrm{~nm}$ and $520 \pm 5 \mathrm{~nm}$. Absorption spectra of perovskite thin films were obtained using ultraviolet-visible light (UV-vis) spectrometer (Shimadzu, Model UV-2450).

\section{Raman spectroscopy}

The Raman vibration modes were measured using Alpha 300R (WITec focus innovations) with excitation wavelength of $532 \mathrm{~nm}$ and $37 \mu \mathrm{W}$ continuous wave laser at room temperature. The microscope objective has a numerical aperture NA $=0.8$ (Olympus 100x). The Raman microscope was operated at the diffraction limit with a laser spot size of less than $1 \mu \mathrm{m}$. The measurements were carried out in backscattering geometry.

\section{X-ray photoemission and Ultraviolet photoelectron spectroscopy (XPS and UPS)}

XPS experiments were carried out by using X-ray source of Magnesium Ka with typical excitation energy output of $1254 \mathrm{eV}$. UPS measurements were performed using a VG ESCA Lab system, an ultrahigh vacuum (UHV) system with a typical base pressure in the range of $\sim 10^{-10}$ mbar equipped with a He discharge lamp. The UV source was an unfiltered He I $(21.2 \mathrm{eV})$ excitation and the sample was biased at $-5 \mathrm{~V}$ to extract the low-energy secondary cutoff. The UV light spot size on the sample is about $1 \mathrm{~mm}$ in diameter.

\section{X-ray absorption spectroscopy (XAS)}

The XAS measurements were performed at the SINS beamline of Singapore Synchrotron Light Source equipped with a Scienta R4000 electron energy analyzer. The samples discussed here were deposited in a separate preparation chamber and they were transferred ex-situ to the SINS beamline. ${ }^{1}$ Prior to the synchrotron measurements, all the samples were annealed to minimize adsorbed contaminants. All spectroscopic measurements were carried out in an ultrahigh vacuum (UHV) chamber with a base pressure of $1 \times 10^{-10} \mathrm{mbar}$. Throughout the experiments, the incident photon energy was initially calibrated using a sputter-cleaned gold foil in electrical contact with the sample. The energy spectra were referenced to the $\mathrm{Au} 4 \mathrm{f}_{7 / 2}$ core level peak at $84.0 \mathrm{eV}$. The $\mathrm{C}$ K-edge XAS spectra were measured in total electron yield (TEY) mode by collecting the sample current with a photon energy resolution of $200 \mathrm{meV}$. The linear polarization factor of the X-ray beam was determined to be more than $90 \%$. All XAS spectra were first normalized to the incident photon intensity $\left(\mathrm{I}_{0}\right)$ monitored by the refocusing mirror. 


\section{Resonant photoemission (RPES)}

RPES spectra were collected at the valence band region with photon energy swept from 280 to $290.3 \mathrm{eV}$ across the $\mathrm{C} 1 \mathrm{~s} \rightarrow \pi^{*}$ resonances. Thus, the spectra were plotted on a binding energy scale with respect to the substrate Fermi level $\left(\mathrm{E}_{\mathrm{F}}\right)$. The presented spectra have been normalized using the incident photon intensity.

\section{Spectroscopic Ellipsometry (SE)}

Spectroscopic ellipsometry measurements were done from 0.6 to $5.0 \mathrm{eV}$ using a commercial J.A. Woollam V-VASE ellipsometer with a compensator and rotating analyzer. The ellipsometer is also fitted with a focusing probe to reduce the beam spot down to $0.4 \mathrm{~mm}$ diameter. We measured the samples using three different incident angles $(\theta)$ at $50^{\circ}, 60^{\circ}$, and $70^{\circ}$ from the sample surface normal. At $70^{\circ}$ the beam spot is increased to $1 \mathrm{~mm}$ x $0.4 \mathrm{~mm}$ elliptical shape due to the grazing angle with respect to the surface. The measurement mode is isotropic with depolarization and the results are analyzed using the J.A. Woollam CompleteEASE software. The raw data, which consists of amplitude ratio $\Psi(\omega)$ and the phase difference $\Delta(\omega)$ between the incident and reflected light, was fitted using three PSEMI-Tri Oscillators and an additional angular spread to the uneven surfaces of the sample. The model is fitted with a using a linear regression method, giving a root mean square (RMS) error of 7.73. Details on the fitting procedures and results are available in the supplementary figures and supplementary notes.

\section{Density Functional Theory (DFT)}

DFT calculation was done using Vienna Ab-Initio Simulation Package (VASP) ${ }^{2-4}$. To correctly simulate experimental band gap and exchange coupling constant, we used DFT with on-site Coulomb interaction (DFT+U) using PBE Generalized Gradient Approximation (GGA-PBE) and the Projector Augmented Wave (PAW) methods ${ }^{5-7}$. The on-site Coulomb interaction $U$ was set as $U=7 \mathrm{eV}$. This value was determined by comparing experimental and calculated exchange coupling constant $J / k_{B}{ }^{8}$. Linear Response was calculated from the converged ground-state using Random Phase Approximation (RPA) method without any local field effects ${ }^{9}$. Reflectance and linear dichroism is calculated using Fresnel equation for anisotropic systems ${ }^{10}$. The energy cutoff was $500 \mathrm{eV}$ with a fine Gamma-centered Monkhorst Pack grid. The crystal structure was based on experimental data ${ }^{11}$ and is relaxed up to $F=-0.02 \mathrm{eV}^{-1}$. 


\section{Figures}

\section{XRD measurements}

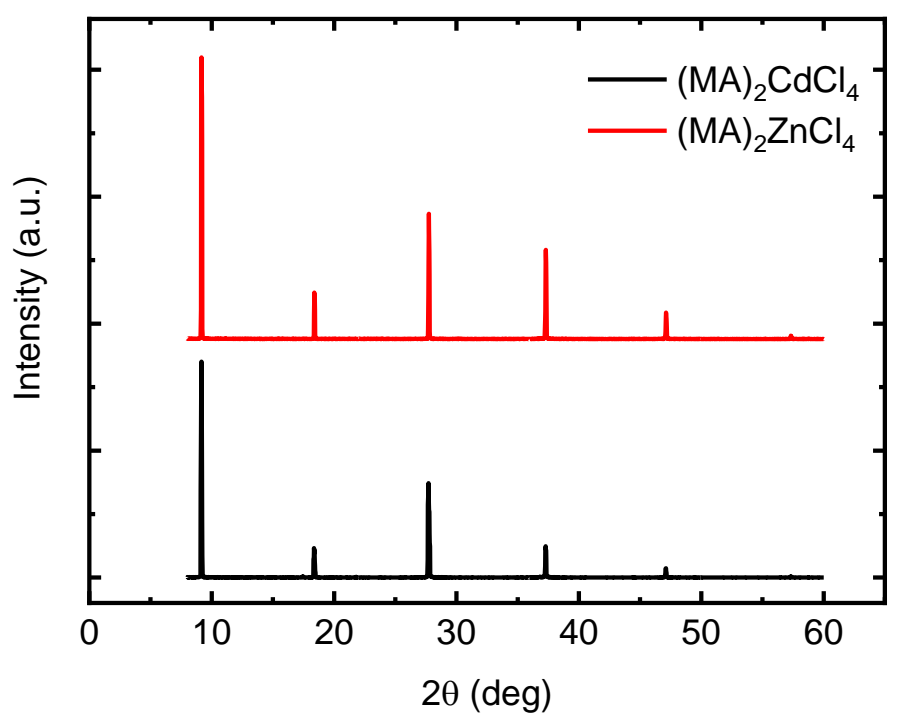

Figure S1. XRD profile of $(\mathrm{MA})_{2} \mathrm{CdCl}_{4}$ and $(\mathrm{MA})_{2} \mathrm{ZnCl}_{4}$.

\section{Topographic Features from AFM and SEM}
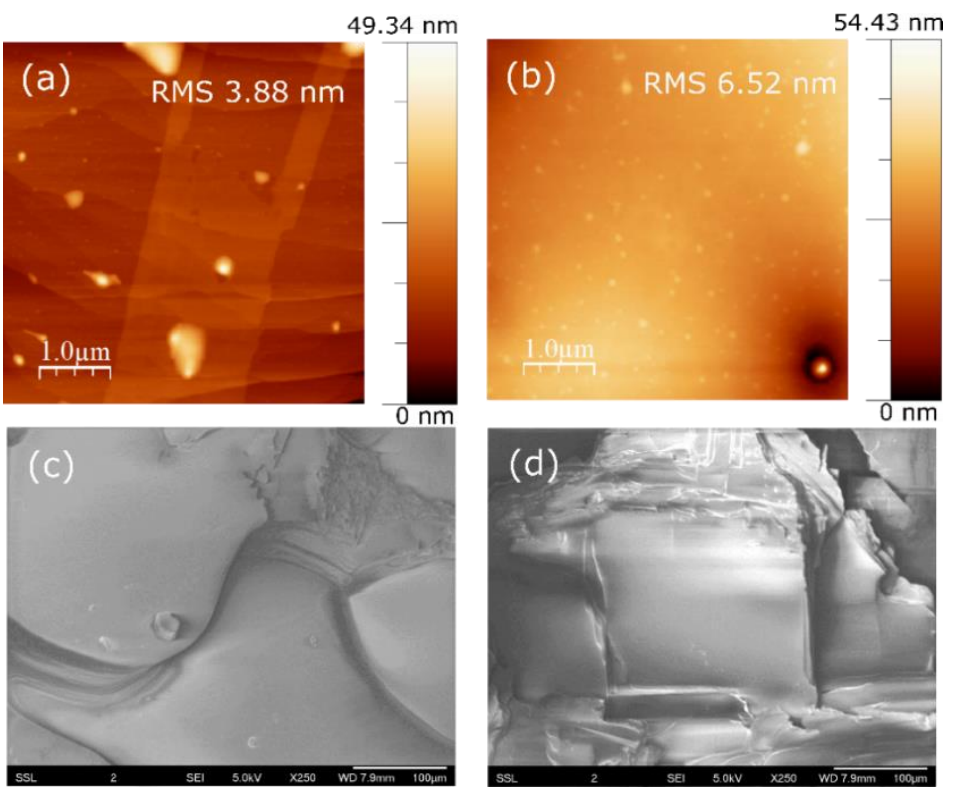

Figure S2. Micrograph features of $(\mathrm{MA})_{2} \mathrm{BCl}_{4}(\mathrm{~B}=\mathrm{Cu}, \mathrm{Cd}, \mathrm{Zn})$ perovskite. (a) AFM tapping mode of (MA) $)_{2} \mathrm{CdCl}_{4}$ and (b) $(\mathrm{MA})_{2} \mathrm{ZnCl}_{4}$ with their respective surface roughness values. The 
scanning size is $5 \times 5 \mu \mathrm{m}^{2}$. SEM images of (d) $(\mathrm{MA})_{2} \mathrm{CdCl}_{4}$ and (e) (MA) $\mathrm{ZnCl}_{4}$. Scale bar as indicated in the respective images.

\section{Stokes Shift from Photoluminescence and Absorption spectra}

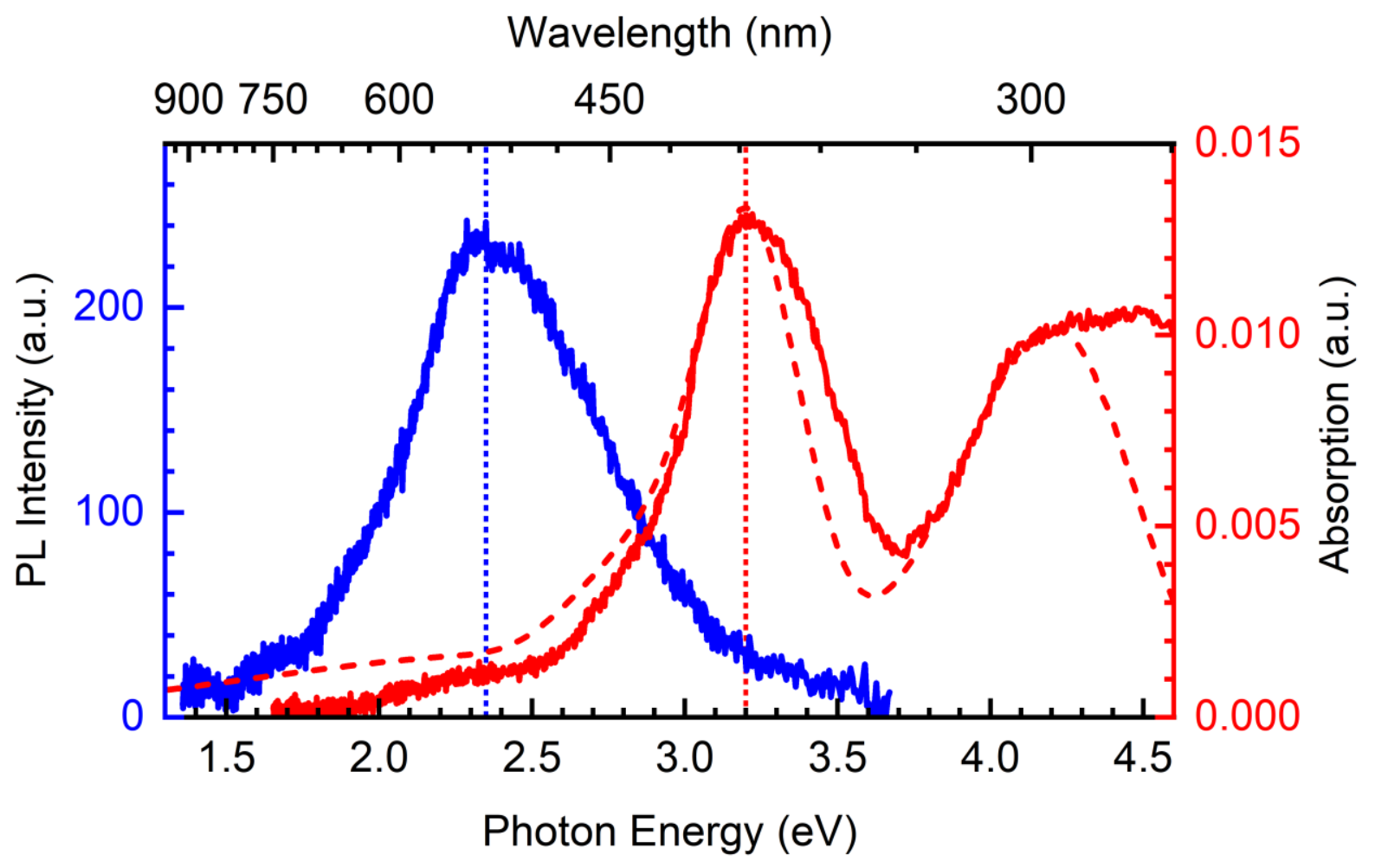

Figure S3. Combined Photoluminescence (blue), experimental absorption spectroscopy (red), and absorption coefficient $\alpha(\omega)$ generated from spectroscopic ellipsometry (red dashed). The experimental absorption has been scaled to fit the absorption coefficient $\alpha(\omega)$. Difference between experimental and SE-generated absorption is due to recombination losses. The calculated Stoke shift is $\approx 140.16 \mathrm{~nm}(\approx 0.83 \mathrm{eV})$. 
Photoluminescence $(\mathrm{MA})_{2} \mathrm{XCl}_{4}(\mathrm{X}=\mathrm{Cu}, \mathrm{Cd}, \mathrm{Zn})$

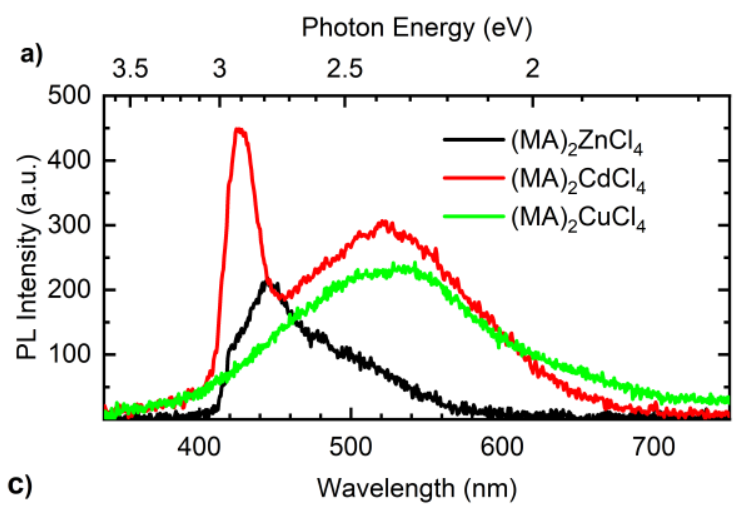

b)
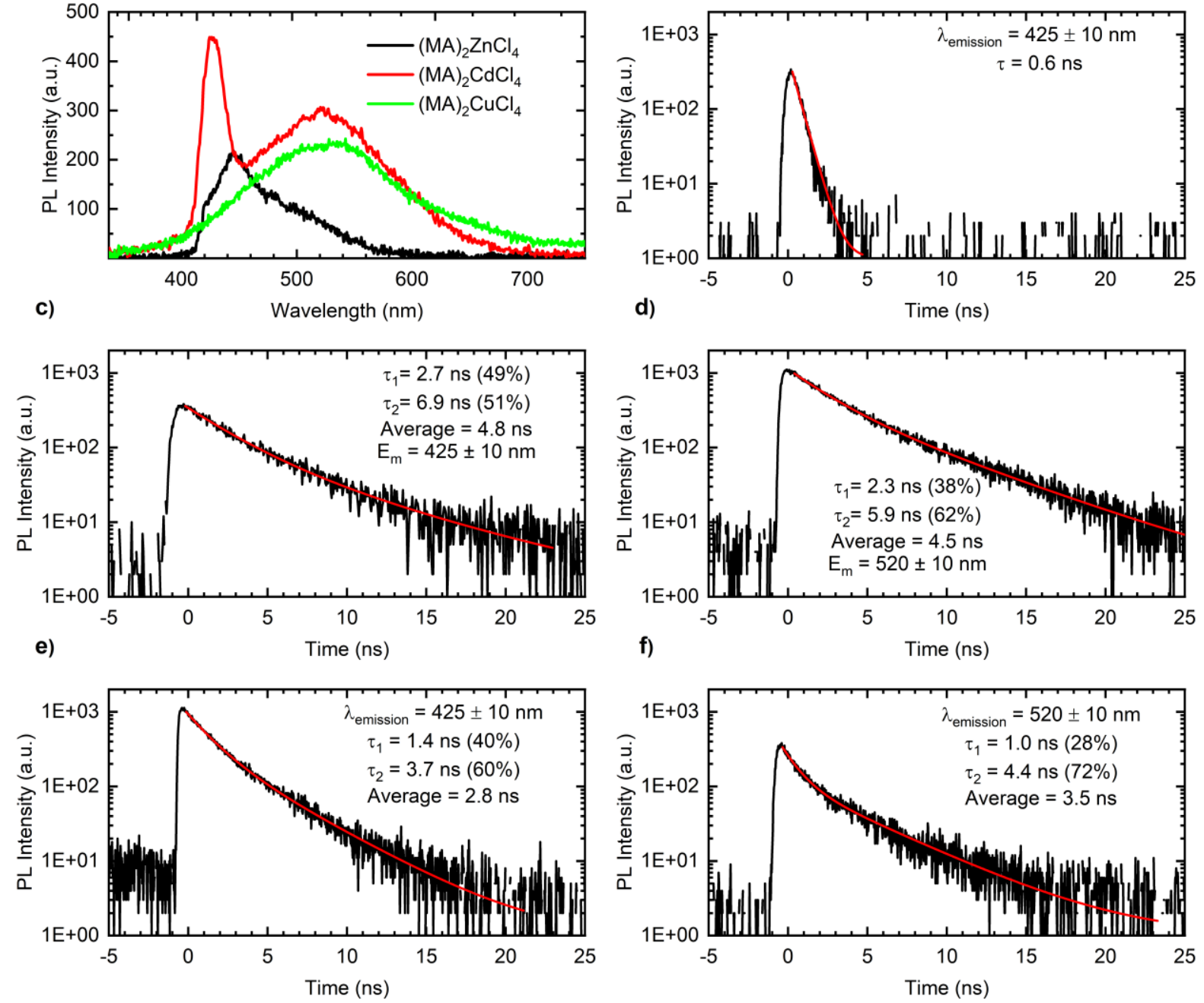

Figure S4. Steady-state photoluminescence and time-resolved PL spectra of hybrid organicinorganic perovskites. (a) PL spectra of (MA) ${ }_{2} \mathrm{ZnCl}_{4},(\mathrm{MA})_{2} \mathrm{CdCl}_{4}$, and (MA) ${ }_{2} \mathrm{CuCl}_{4}$. (b) TR-PL spectra of $(\mathrm{MA})_{2} \mathrm{ZnCl}_{4}$ with emission wavelength at $425 \mathrm{~nm}$. (c-d) $(\mathrm{MA})_{2} \mathrm{CuCl}_{4}$ with emission wavelength at $425 \mathrm{~nm}$ and $520 \mathrm{~nm}$. (e-f) (MA) ${ }_{2} \mathrm{CdCl}_{4}$ with emission wavelength at $425 \mathrm{~nm}$ and $520 \mathrm{~nm}$. Red lines indicate mono/biexponential fitting of the raw TR-PL spectra (black). The extracted short and long component lifetime are shown in the respective inset. 
Low temperature Photoluminescence measurement

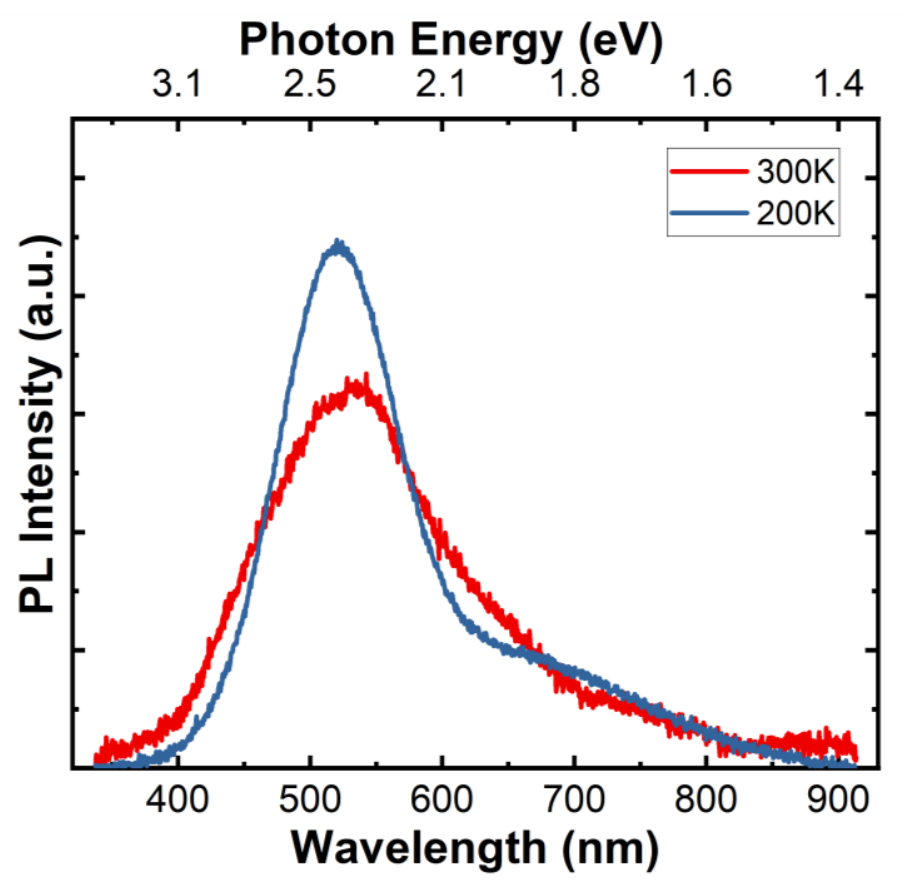

Figure S5. Photoluminescence of $(\mathrm{MA})_{2} \mathrm{CuCl}_{4}$ at room temperature $(300 \mathrm{~K})$ and low temperature (200 K).

\section{X-ray Absorption Spectroscopy of (MA) $)_{2} \mathrm{CdCl}_{4}$}

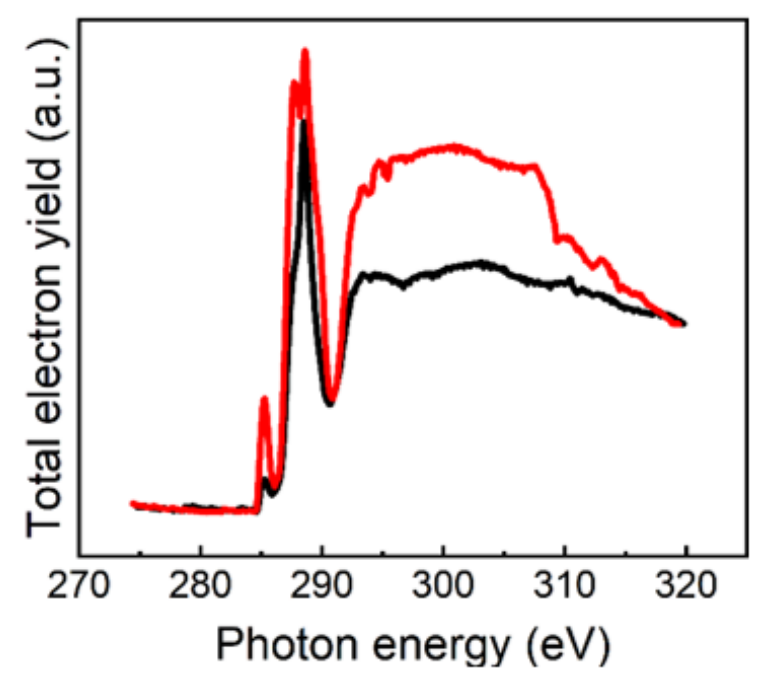


Figure S6. Carbon K-edge XAS spectra of $(\mathrm{MA})_{2} \mathrm{CdCl}_{4}$. The angular dependent measurements were taken with grazing incidence angle $20^{\circ}$ (black) and normal incidence $90^{\circ}$ (red). The estimated average tilt angle is $63.31^{\circ}$.

\section{Resonant Photoemission Spectroscopy (RPES)}

a)

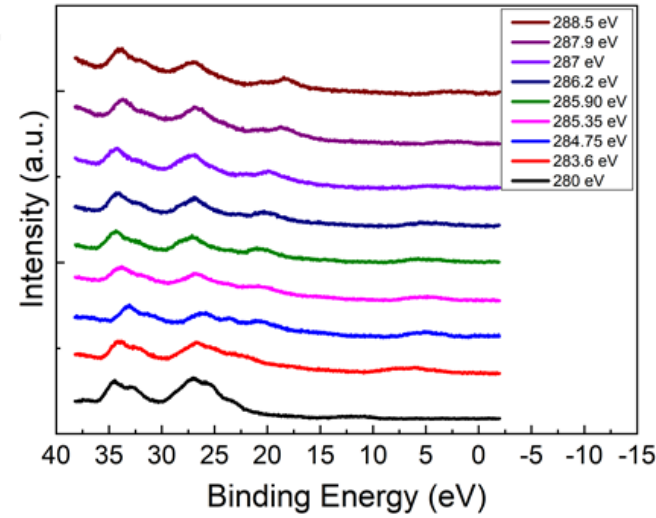

c)

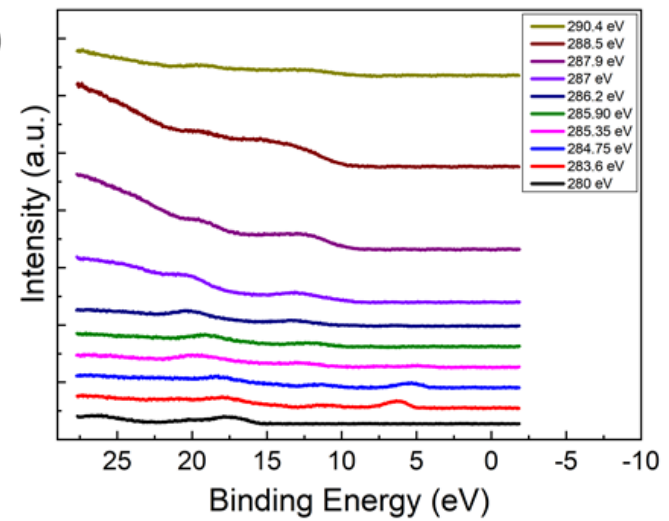

b)

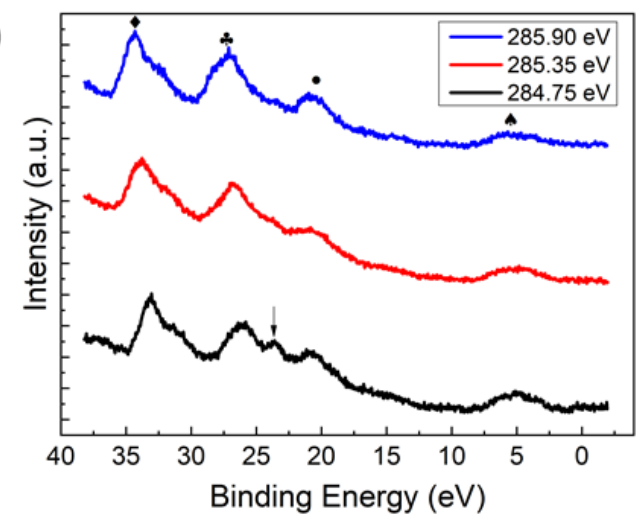

d)

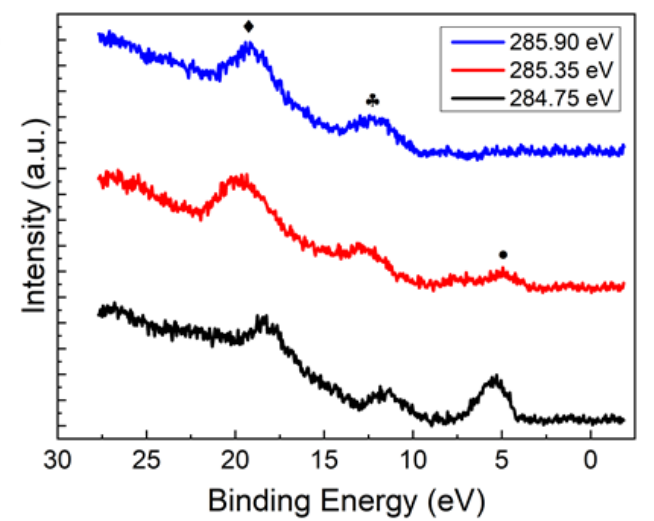

Figure S7. Resonant photoemission spectra of 2D-Hybrid Organic-Inorganic Perovskites close to K-edge absorption threshold. (a-c) Full scale region RPES of (MA) ${ }_{2} \mathrm{CuCl}_{4},(\mathrm{MA})_{2} \mathrm{ZnCl}_{4}$ and $(\mathrm{MA})_{2} \mathrm{CdCl}_{4}$. (d-f) Selected RPES region close to the on-resonant transition regime. 


\section{Absorption Coefficient and Tauc Plot}
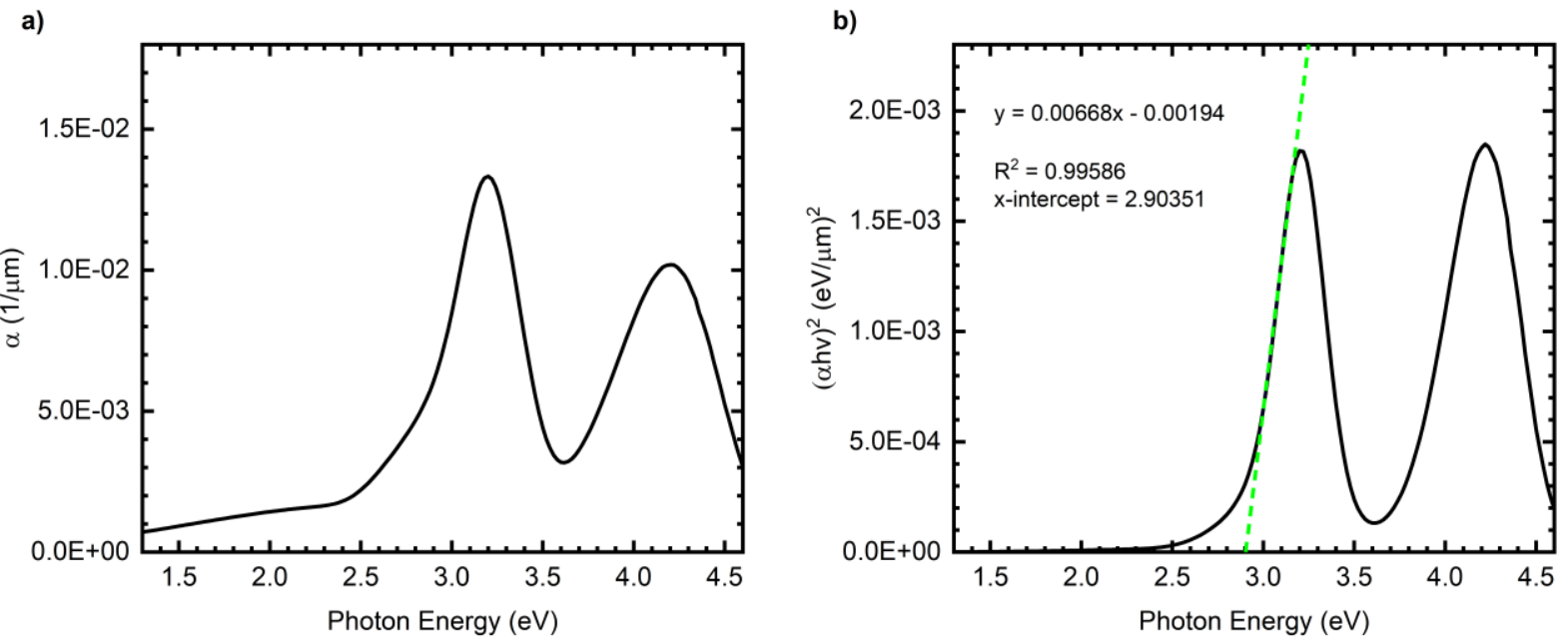

Figure S8. a) Absorption coefficient $(\alpha(\omega))$ and b) Tauc plot of direct allowed transitions $\left((\alpha h v)^{2}\right)$ calculated from $\tilde{\varepsilon}(\omega)$ (see supplementary notes). The optical bandgap estimated from Tauc plot is $2.91 \mathrm{eV}$. The onset of absorption is estimated to be $2.36 \mathrm{eV}$. 


\section{Loss Function Fitting}
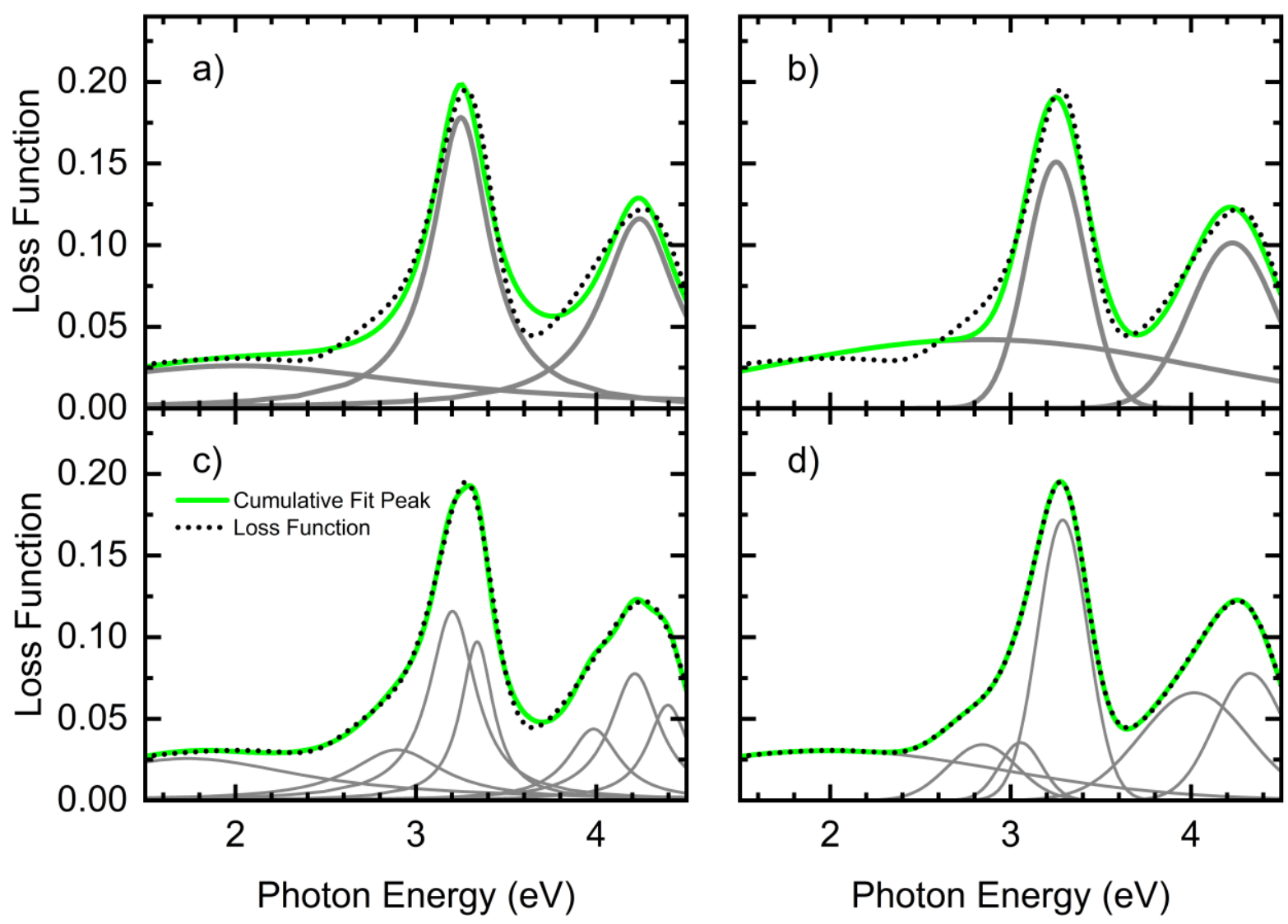

Figure S9. Fitting for loss function $\left(-\operatorname{Im}[\varepsilon(\omega)]^{-1}\right)$ using a) single peak Lorentzian function, b) single peak Gaussian function, c) multi-peaks Lorentz function, and d) multi-peaks Gaussian function. Skewness of peak B' $(4.26 \mathrm{eV})$ using data points between $3.8 \mathrm{eV}-4.7 \mathrm{eV}$ is -0.53 . 


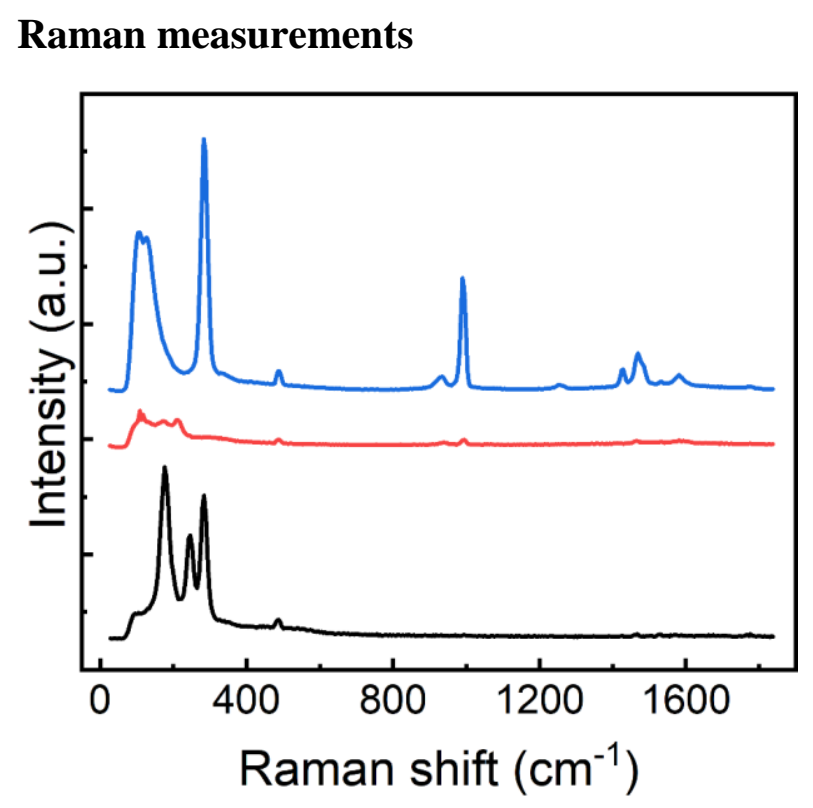

Figure S10. Raman profiles of (MA) ${ }_{2} \mathrm{ZnCl}_{4}$ (blue), (MA) $)_{2} \mathrm{CdCl}_{4}$ (red), and (MA) ${ }_{2} \mathrm{CuCl}_{4}$ (black). 
XPS core levels spectra
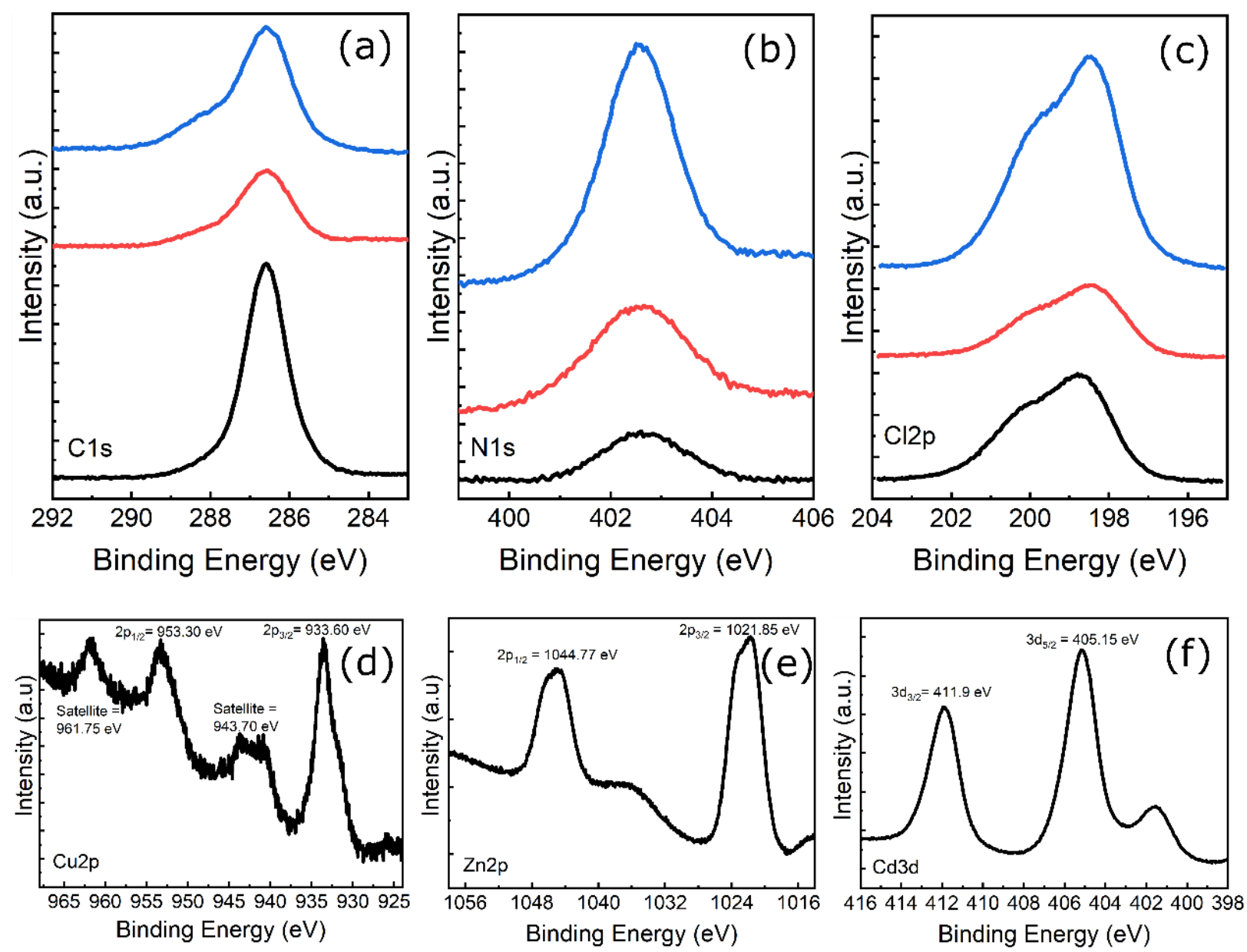

Figure S11. X-ray photoemission spectra of $(\mathrm{MA})_{2} \mathrm{BCl}_{4}(\mathrm{~B}=\mathrm{Cu}, \mathrm{Cd}, \mathrm{Zn})$ perovskites. (a) $\mathrm{C} 1 \mathrm{~s}$ core level represents main prominent peak at $286.6 \mathrm{eV}$. (b) $\mathrm{N} 1 \mathrm{~s}$ core level concentrated at 402.6 $\mathrm{eV}$. (c) $\mathrm{Cl} 2 \mathrm{p}$ core level exhibited with two components demonstrating the occurrence of $\mathrm{Cl} 2 \mathrm{p}_{3 / 2}$ and $\mathrm{Cl} 2 \mathrm{p}_{1 / 2}$. (MA) ${ }_{2} \mathrm{CuCl}_{4}$ (black), (MA) $\mathrm{ZnCl}_{4}$ (red) and (MA) $\mathrm{CdCl}_{4}$ (blue). The respective contributed B-site metal (d) $\mathrm{Cu} 2 \mathrm{p}$ core level, (e) $\mathrm{Zn} 2 \mathrm{p}$ core level and (f) $\mathrm{Cd} 3 \mathrm{~d}$ core level. 
XPS Cu 2p3/2 deconvolution peak and $\mathrm{Cu}$ composition

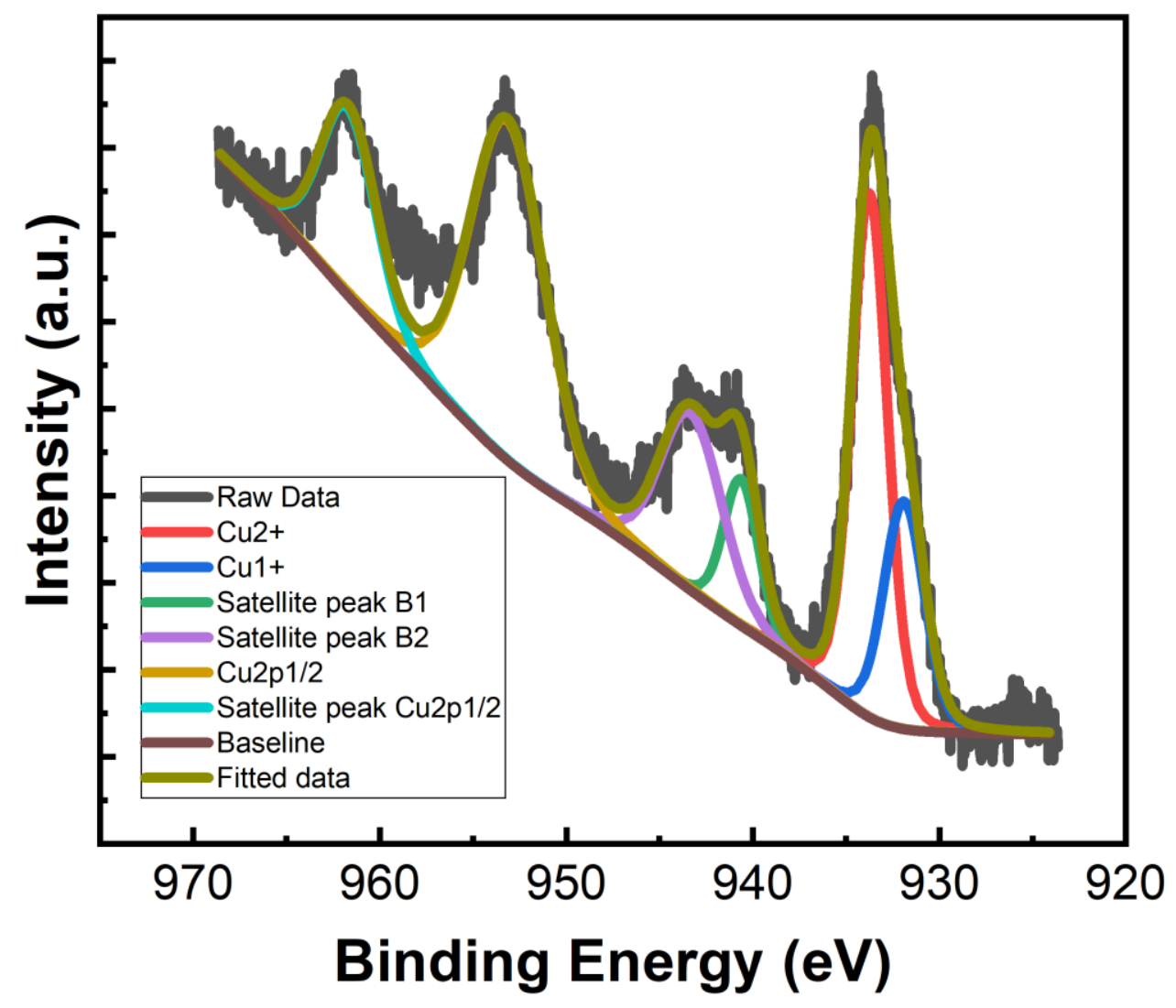

Figure S12. XPS $\mathrm{Cu} 2 \mathrm{p}$ core level deconvolution peaks using CasaXPS software. Each of deconvoluted peaks are represents with different colors. The extracted peaks are $933.75 \mathrm{eV}\left(\mathrm{Cu}^{2+}\right)$, $931.9 \mathrm{eV}\left(\mathrm{Cu}^{+}\right), 940.7 \mathrm{eV}$ (satellite peak B1), $943.55 \mathrm{eV}$ (satellite peak B2), $953.25 \mathrm{eV}\left(\mathrm{Cu} 2 \mathrm{p}_{1 / 2}\right)$, and $961.9 \mathrm{eV}$ (satellite peak $\mathrm{Cu} 2 \mathrm{p}_{1 / 2}$ ). 


\section{Spectroscopic Ellipsometry Optical Model Fitting}

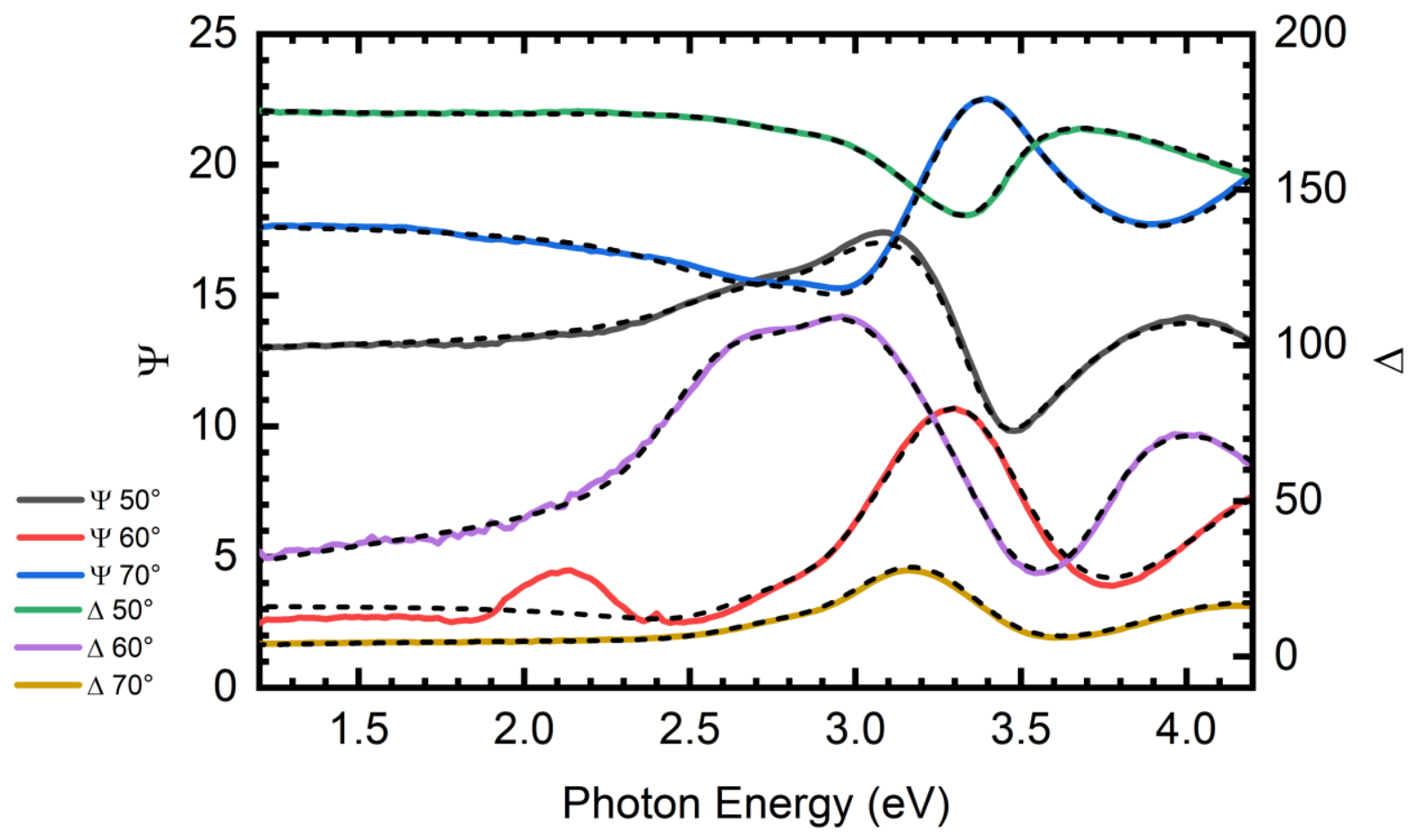

Figure S13. Fitting of optical models generated from PSEMI-TRI functions. $\Psi(\omega)$ and $\Delta(\omega)$ correspond to amplitude ratio and phase difference between incident and reflected light. The sample is modeled as a substrate with additional angular spread of the reflected light by $4.35 \%$. The optical model is based on the sample's uneven surfaces. The root mean square error of the fitting is 6.063. The large fitting error observed in $60^{\circ}$ is due to Brewster's angles (see supplementary notes). 


\section{Reflectance and Linear Dichroism using DFT-generated $\tilde{\varepsilon}(\omega)$}

a)

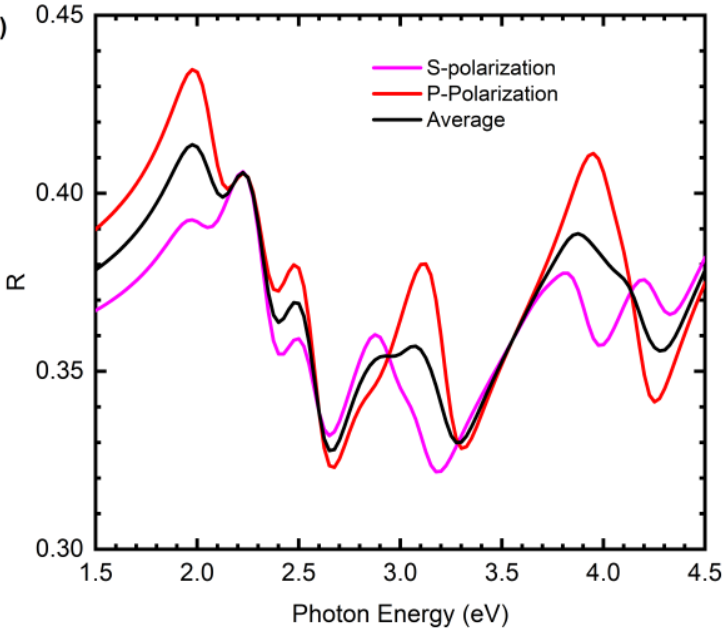

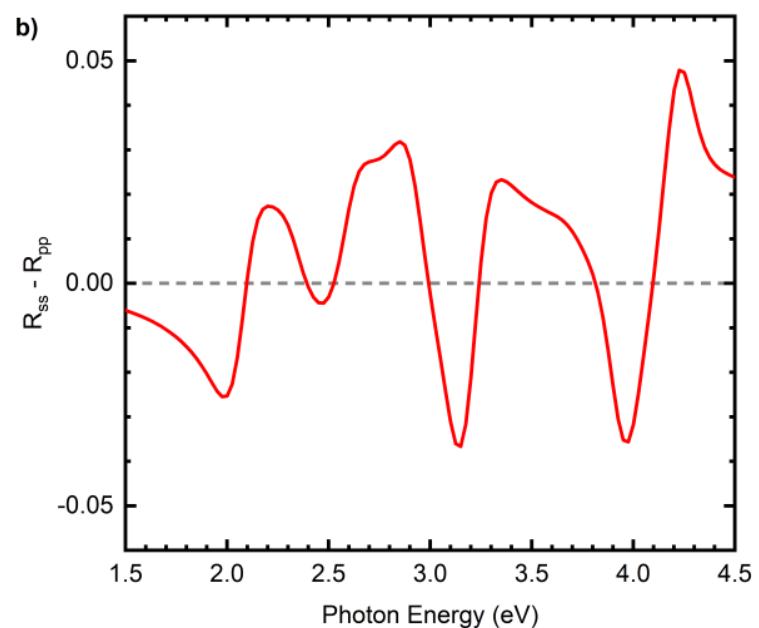

Figure S14. a) Reflectance at normal incidence and b) linear dichroism of (MA) ${ }_{2} \mathrm{CuCl}_{4} \cdot \mathrm{R}_{\mathrm{ss}}$ denotes the reflectance of s-polarized light from s-polarized incident light. Similarly, $\mathrm{R}_{\mathrm{pp}}$ denotes the reflectance of p-polarized light from p-polarized incident light. The linear dichroism is generated from the difference between $R_{s s}$ and $R_{p p}$ and is normalized for clarity. The reflectance is based on complex dielectric function $\tilde{\varepsilon}(\omega)$ calculated using RPA without local-field effects (see methods section). 


\section{Tables}

Table S1. The extracted resonant transition peaks of perovskites acquired from C-K edge XAS spectra

\begin{tabular}{|l|l|l|l|l|l|}
\hline Perovskites & $\boldsymbol{\pi}_{\mathbf{1}} *(\mathbf{e V})$ & $\boldsymbol{\pi}_{\mathbf{2}}^{*}(\mathbf{e V})$ & $\boldsymbol{\pi}_{\mathbf{3}} *(\mathbf{e V})$ & $\boldsymbol{\sigma}_{1} *(\mathbf{e V})$ & $\boldsymbol{\sigma}_{\mathbf{2}} *(\mathbf{e V})$ \\
\hline$(\mathrm{MA})_{2} \mathrm{CuCl}_{4}$ & 285.3 & 287.8 & 288.8 & 293.8 & 300.8 \\
\hline$(\mathrm{MA})_{2} \mathrm{CdCl}_{4}$ & 285.3 & 287.8 & 288.5 & 293.4 & 294.7 \\
\hline$(\mathrm{MA})_{2} \mathrm{ZnCl}_{4}$ & 285.3 & 287.7 & 288.6 & 293.3 & 294.7 \\
\hline
\end{tabular}

Table S2. Loss function fitting using single-peak Lorentzian function

\begin{tabular}{|l|l|l|l|l|}
\hline $\begin{array}{c}\text { Corresponding } \\
\text { LF Peak }\end{array}$ & \multicolumn{1}{|c|}{ Energy (eV) } & FWHM & $\begin{array}{c}\text { Dephasing Time } \\
\text { (fs) }\end{array}$ & $\begin{array}{c}\text { Free Electron } \\
\text { Scattering (eV) }\end{array}$ \\
\hline Peak A' & 3.25 & 0.38 & 3.43 & 0.77 \\
\hline Peak B' & 4.24 & 0.51 & 2.60 & 1.01 \\
\hline
\end{tabular}

Table S3. Loss function fitting using single-peak Gaussian function

\begin{tabular}{|l|l|l|l|l|}
\hline $\begin{array}{c}\text { Corresponding } \\
\text { LF Peak }\end{array}$ & \multicolumn{1}{|c|}{ Energy (eV) } & FWHM & $\begin{array}{c}\text { Dephasing Time } \\
\text { (fs) }\end{array}$ & $\begin{array}{c}\text { Free Electron } \\
\text { Scattering (eV) }\end{array}$ \\
\hline Peak A' & 3.25 & 0.38 & 3.38 & 0.77 \\
\hline Peak B' & 4.24 & 0.56 & 2.36 & 1.02 \\
\hline
\end{tabular}

Table S4. Loss function fitting using multi-peak Lorentzian function

\begin{tabular}{|l|l|l|l|l|}
\hline $\begin{array}{c}\text { Corresponding } \\
\text { LF Peak }\end{array}$ & Energy (eV) & FWHM & $\begin{array}{c}\text { Dephasing Time } \\
\text { (fs) }\end{array}$ & $\begin{array}{c}\text { Free Electron } \\
\text { Scattering (eV) }\end{array}$ \\
\hline Peak A' & 3.20 & 0.32 & 4.17 & 0.63 \\
\hline Peak A' & 3.34 & 0.22 & 5.99 & 0.44 \\
\hline Peak B' & 3.98 & 0.35 & 3.80 & 0.69 \\
\hline Peak B' & 4.21 & 0.33 & 3.94 & 0.67 \\
\hline Peak B' & 0.28 & 4.77 & 0.55 \\
\hline Average for Peak A' & 5.40 & 4.08 & 0.54 \\
\hline Average for Peak B' & 4.17 & 0.63 \\
\hline
\end{tabular}

Table S5. Loss function fitting using multi-peak Gaussian function

\begin{tabular}{|l|l|l|l|l|}
\hline $\begin{array}{c}\text { Corresponding } \\
\text { LF Peak }\end{array}$ & \multicolumn{1}{|c|}{ Energy (eV) } & FWHM & $\begin{array}{c}\text { Dephasing Time } \\
\text { (fs) }\end{array}$ & $\begin{array}{c}\text { Free Electron } \\
\text { Scattering (eV) }\end{array}$ \\
\hline Peak A' & 3.29 & 0.33 & 4.04 & 0.65 \\
\hline Peak B' & 4.01 & 0.70 & 1.88 & 1.40 \\
\hline
\end{tabular}




\begin{tabular}{|c|c|c|c|c|}
\hline Peak B' & 4.32 & 0.46 & 2.88 & 0.91 \\
\hline \multicolumn{3}{|c|}{ Average for Peak B' } & 2.38 & 1.15 \\
\hline
\end{tabular}




\section{Supplementary Notes}

\section{XAS Orientation Linear Dichroism}

Since the intensity differences are indicative of orientation linear dichroism, one should consider that the cross sectional of the resonant photo-excitation process is highly dependent on the relative orientation of the electric-field vector $(\vec{E})$ of the incident synchrotron light with respect to the transition dipole moment (TDM) of the active material ${ }^{12}$. Due to correlated transition resonance states, one could determine that the resonant excitation arises from the ground state (1s) to an unoccupied state where a resonant intensity is strongly sensitive to the $\vec{E}$ vector. In other words, the XAS intensity is strongly enhanced when $\vec{E}$ is parallel to the governing orbital sample vector.

From the difference of profiles from angle-dependent XAS measurements, the average tilt angle of MA moiety could be estimated using angular dichroism relationship based on the following equation ${ }^{13-14}$ :

$$
\frac{I_{90^{0}}}{I_{20^{0}}}=\frac{1}{2} \tan ^{2} \theta
$$

Where $I_{90^{\circ}}$ is the intensity at $90^{\circ}, I_{20^{\circ}}$ is the intensity at $20^{\circ}$, and $\theta$ is the average tilt angle.

\section{XPS Cu 2p3/2 deconvolution peak and Cu composition}

Using core level peak fitting software such as casaXPS, the ratio of $\mathrm{Cu}^{2+}$ and $\mathrm{Cu}^{+}$can be determined based on the following equation:

$\mathrm{n}\left(\mathrm{Cu}^{2+}\right)=\frac{B+A 1}{A+B} \times 100$

$\mathrm{n}\left(\mathrm{Cu}^{+}\right)=\frac{A 2}{A+B} \times 100=\frac{A-A 1}{A+B} \times 100$

Where $\mathrm{B}$ is the total area of both satellite peaks next to $\mathrm{Cu} 2 \mathrm{p}_{3 / 2}$ peak, $\mathrm{A} 1$ is the integrated area of the $\mathrm{Cu}^{2+}$ main peak, and $\mathrm{A} 2$ is the $\mathrm{Cu}^{+}$main peak. In addition, $\mathrm{A}$ is the total area of the $\mathrm{Cu} 2 \mathrm{p}_{3 / 2}$ peak, including the area of both $\mathrm{Cu}^{2+}$ and $\mathrm{Cu}^{+}$species.

To indicate any chemical interaction between the MA moieties and the inorganic elements, we sweep the core level of carbon $1 s$ to assess the sample chemical compositions. Figure S10 shows that the evolution of $\mathrm{C} 1 \mathrm{~s}$ core level state is centered at $286.60 \mathrm{eV}$. This prominent peak is typically found in most of HOIPs originating from the $\mathrm{C}-\mathrm{N}$ bonding signature. To substantiate the finding, the $\mathrm{N}$ 1s core level at $402.60 \mathrm{eV}$ and $\mathrm{Cl} 2 \mathrm{p}$ also display the expected respective signals at and $2 \mathrm{p}_{3 / 2}$ $=198.50 \mathrm{eV} ; 2 \mathrm{p}_{1 / 2}=200.10 \mathrm{eV}$ as shown in Figure S9b and c, respectively. Figure S9d displays the $\mathrm{Cu} 2 \mathrm{p}$ core level across the binding energy of $925-965 \mathrm{eV}$, indicating the presence of $\mathrm{Cu}^{2+}$ 
oxidation state. Here, the two peaks centered at $933.60 \mathrm{eV}$ and $953.30 \mathrm{eV}$ correspond to $2 \mathrm{p}_{3 / 2}$ and $2 \mathrm{p}_{1 / 2}$ core levels, respectively. In addition, the accompanying satellite peaks (943.70 and 961.75 $\mathrm{eV})$ are also observed. We also note the presence of $\mathrm{Zn} 2 \mathrm{p}$ and $\mathrm{Cd} 3 \mathrm{~d}$ core levels at their respective samples as displayed in Figure S9e and f, respectively. Peak fitting of the $\mathrm{Cu} 2 \mathrm{p}_{3 / 2}$ peaks revealed that the atomic ratio analysis exhibited a composition profile of approximately $79.07 \% \mathrm{Cu}^{2+}$ and $20.93 \% \mathrm{Cu}^{+}$(Figure $\mathrm{S} 10$ ). This strengthen the hypothesis that the chemical state of $\mathrm{Cu}^{2+}$ serve as the prominent governing oxidation state in this compound.

\section{D Raman Spectra}

Raman measurement is performed to complement the previous PL characterization in order to identify the presence of the contributing molecular groups of 2D-HOIPs as presented in Figure S4. The Raman spectrum shows three fingerprint frequency regions: a) $\mathrm{Cu}-\mathrm{Cl}$ vibration modes under $350 \mathrm{~cm}^{-1}$ that indicates the presence of the $\mathrm{CuCl}_{4}{ }^{2-}$ anion; b) $\mathrm{CH}_{2}, \mathrm{NH}_{3}$ and $\mathrm{C}-\mathrm{N}-\mathrm{H}$ vibrations modes between 350 and $1600 \mathrm{~cm}^{-1}$; c) C-H stretching vibrations, which were observed above 2500 $\mathrm{cm}^{-1}$. According to these vibration signatures, we confirmed the presence of 2D nature of assynthesized $(\mathrm{MA})_{2} \mathrm{CuCl}_{4}$.

\section{Spectroscopic Ellipsometry (SE) Fitting}

As discussed in the method section, we conducted the ellipsometry measurement at three different incident angles: $50^{\circ}, 60^{\circ}$, and $70^{\circ}$ degrees. These angles were selected to be near the Brewster's angle $\theta_{B}$ where the difference between $\mathrm{p}$ - and s-polarized light is maximum. In addition, pseudoBrewster angle $\theta_{B}^{\prime}$ is the incident angle when p-polarized light is minimum/near zero. The angle is dependent on the complex refractive index $(N=n+i k)$ and photon energy, where for most materials $\theta_{B}$ ranges between $60^{\circ}-80^{\circ}$ degrees. Since SE measures the amplitude ratio between the p-polarized light and s-polarized light $\left(\rho=\tan (\Psi) \exp (\mathrm{i} \Delta)=\frac{R_{P}}{R_{S}}\right)$, the measurements done near $\theta_{B}$ or $\theta_{B}^{\prime}$ will be more accurate ${ }^{10}$. The Brewster's angle can be calculated using Snell's law with the relation below:

$$
\tan \theta_{B}=\frac{N_{t}}{N_{i}}
$$

Where $N_{t}$ and $N_{i}$ are the complex refractive index of the studied sample and air, respectively. However, incident angles very near $\theta_{B}{ }^{\prime}$ may become inaccurate. If the detected p-polarized light intensity is small or undetectable, the measured $R_{P}$ will fluctuate rapidly and reduce the accuracy of the measurement. In this study, we observe very accurate data for incident angles $50^{\circ}$ and $80^{\circ}$ degrees, but a broad bump is seen at $\approx 2.1 \mathrm{eV}$ for $60^{\circ}$ degrees incident angle (Fig. S13). We examined the $\rho(\Psi, \Delta)$ values to determine whether the bump is caused by the Brewster's angle of $(\mathrm{MA})_{2} \mathrm{CuCl}_{4}$ or other sources, e.g. surface features/defects. Here, we observe that the $\Psi(\omega)$ is minimum $(\sim 4.52)$ at $60^{\circ}$ degrees incident angle. $\Delta(\omega)$ shows a $180^{\circ}$ jump between $70^{\circ}$ degrees 
incident angle to $50^{\circ}$ degrees incident angle. These measurements indicate that the Brewster's angle is located at $60^{\circ}$ degrees incident angle, where the p-polarized intensity is minimum between 1.18 and $2.35 \mathrm{eV}$. In order to compensate the inaccuracy at this energy range, we initially fit the other two incident angles and use it as a base to fit the whole dataset. Despite the inaccuracy of the fit at below $2.35 \mathrm{eV}$, we found that at higher energies the optical model fits the ellipsometric data very well (Fig. S11)

For the optical model to fit the ellipsometric dataset, we use an isotropic optical model of a substrate with uniform thickness. Thus, the data taken at the three incident angles are correspond to the isotropic dielectric function. In our model, we utilized the three (3) PSEMI-Tri Oscillators derived from the Herzinger-Johs Psemi function ${ }^{15}$. These oscillators are then fitted using CompleteEASE® software via a linear regression method. All oscillators are Kramers-Kronig consistent ${ }^{16}$. The fitting results can be seen in the supplementary material Fig S11.

After extracting the dielectric function, we calculate the reflectivity at normal incidence, absorption coefficient, and loss function using these relations ${ }^{10}$ :

$$
\begin{gathered}
n=\left\{\frac{\left[\varepsilon_{1}+\left(\varepsilon_{1}^{2}+\varepsilon_{2}^{2}\right)^{\frac{1}{2}}\right]}{2}\right\}^{\frac{1}{2}} \\
k=\left\{\frac{\left[-\varepsilon_{1}+\left(\varepsilon_{1}^{2}+\varepsilon_{2}^{2}\right)^{\frac{1}{2}}\right]}{2}\right\}^{\frac{1}{2}} \\
R=\frac{(n-1)^{2}+k^{2}}{(n+1)^{2}+k^{2}} \\
\alpha=\frac{4 \pi k}{\lambda} \\
-\operatorname{Im}[\tilde{\varepsilon}]^{-1}=\frac{\varepsilon_{2}}{\varepsilon_{1}^{2}+\varepsilon_{2}^{2}}
\end{gathered}
$$

These equations allow SE to generate and predict other optical experiments with directly measurable properties, such as reflectance, absorption, and electron energy loss spectroscopy (EELS) $^{10,17-18}$.

\section{Loss Function Fitting}

In low-correlated metals, the free electron scattering $(1 / \tau)$ can be estimated using the plasmon energy $\left(\omega_{p}\right)$ and zero-crossing of $\varepsilon_{1}{ }^{19}$ : 


$$
\frac{1}{\tau}=\sqrt{\omega_{p}-\omega_{\varepsilon_{1}=0}}
$$

However, since correlated plasmons do not possess a zero-crossing of $\varepsilon_{1}$, we can estimate $1 / \tau$ and plasmons dephasing time ( $\left.T_{2}\right)$ using the full-width half maximum (FWHM) of the peaks in the loss function ${ }^{17,19-20}$. This can be done with these relations:

$$
\begin{gathered}
\text { FWHM }=2 \hbar \Gamma \\
T_{2}=\frac{1}{\Gamma}=2 \tau
\end{gathered}
$$

where $\Gamma$ denotes the estimated electron scattering rate. We would like to highlight that the estimation of plasmon dephasing time using FWHM analysis and Lorentzian fitting are widely used in plasmonic studies in metals ${ }^{19}$. However, the shapes of peak A' and peak B' do not conform to the Lorentzian function since electrons in (MA) $\mathrm{CuCl}_{4}$ are strongly correlated and confined in a certain direction. This was also observed in correlated plasmons found in inorganic perovskites ${ }^{17}$, ${ }^{20}$. Despite this, using Lorentzian fits reveal new insights on the behavior of correlated plasmons, such as how the plasmons behaves in electron/hole doping in cuprates ${ }^{20}$. Thus, these analyses can be regarded as an estimation of the dephasing time of correlated plasmons and that further studies are required to fully understand correlated plasmon loss and dissipation.

To determine the dephasing time $\left(T_{2}\right)$, we have conducted single peak fitting and multi-peak fitting schemes using Lorentzian and Gaussian function. For single peak fitting, we estimate the broad feature at $\approx 1.8 \mathrm{eV}$, peak A', and peak B' as a single Lorentzian or Gaussian peak (Fig. S9a and $\mathrm{S} 9 \mathrm{~b}$ ). Since single-peak fitting does not reproduce key features in the experimental data, more peak fits are required to correctly model the loss function. For multi-peak fitting, we included additional features, such as the shoulder peak C' and multiple Lorentzian/Gaussian peaks for peak A' and B'.

The details of the fitting results are shown in Table S2-S5. For Lorentzian fitting, we use two Lorentzian peaks for peak A' and three Lorentzian peaks for peak B'. Peak C' and the broad feature in $1.8 \mathrm{eV}$ are modelled as one Lorentzian peak. The average dephasing times for peak A' and B' are 5.08 fs and 4.17 fs. Meanwhile, for Gaussian fitting, we use one Gaussian peak for peak A' and two Gaussian peaks for peak B'. Peak C' and the broad feature in $1.8 \mathrm{eV}$ are modelled as three Gaussian peaks. The average dephasing times for peak A' and B' are 4.03 fs and 2.38 fs. These fitting results have been plotted in Fig. S9.

The average dephasing times of peak A' and peak B' for all fitting modes are $3.98 \mathrm{fs}$ and $2.88 \mathrm{fs}$, respectively. These values are similar to the results of single peak fitting using Lorentzian function ( $3.28 \mathrm{fs}$ and $2.60 \mathrm{fs}$ ). We emphasize again that the dephasing times of correlated plasmons are an estimation. The exact dissipation and losses require further studies. Therefore, we report the dephasing time values of the single Lorentzian peak fit and include the fitting in the supplementary materials (Fig. S9) 


\section{$\underline{\text { References }}$}

1. Cortecchia, D.; Dewi, H. A.; Yin, J.; Bruno, A.; Chen, S.; Baikie, T.; Boix, P. P.; Grätzel, M.; Mhaisalkar, S.; Soci, C., et al. Lead-Free $\mathrm{MA}_{2} \mathrm{CuCl}_{\mathrm{x}} \mathrm{Br}_{4-\mathrm{x}}$ Hybrid Perovskites. Inorg. Chem. 2016, 55 (3), 1044-1052.

2. Kresse, G., and Jürgen Furthmüller. Efficient Iterative Schemes for Ab Initio Total-Energy Calculations using a Plane-Wave Basis Set. Phys. Rev. B 1996, 54, 11169.

3. Hohenberg, P.; Kohn, W. Inhomogeneous Electron Gas. Phys. Rev. 1964, 136 (3B), B864.

4. Kohn, W.; Sham, L. J. Self-Consistent Equations Including Exchange and Correlation Effects. Phys. Rev. 1965, 140 (4A), A1133.

5. Liechtenstein, A.; Anisimov, V.; Zaanen, J. Density-Functional Theory and Strong Interactions: Orbital Ordering in Mott-Hubbard Insulators. Phys. Rev. B 1995, 52 (8), R5467.

6. Blöchl, P. E. Projector Augmented-Wave Method. Physical Review B 1994, 50 (24), 17953.

7. Perdew, J. P.; Burke, K.; Ernzerhof, M. Generalized Gradient Approximation Made Simple. Phys. Rev. Lett. 1996, 77 (18), 3865.

8. Zolfaghari, P.; Wijs, G. A. d.; Groot, R. A. d. The Electronic Structure of OrganicInorganic Hybrid Compounds: $\left(\mathrm{NH}_{4}\right)_{2} \mathrm{CuCl}_{4},\left(\mathrm{CH}_{3} \mathrm{NH}_{3}\right)_{2} \mathrm{CuCl}_{4}$ and $\left(\mathrm{C}_{2} \mathrm{H}_{5} \mathrm{NH}_{3}\right)_{2} \mathrm{CuCl}_{4}$. J. Phys. Condens. Matter 2013, 25 (29), 295502.

9. Ehrenreich, H.; Cohen, M. H. Self-Consistent Field Approach to the Many-Electron Problem. Phys. Rev. 1959, 115 (4), 786.

10. Fujiwara, H. Spectroscopic Ellipsometry: Principles and Applications. Wiley: 2007.

11. Pabst, I.; Fuess, H.; Bats, J. Structure of Monomethylammonium Tetrachlorocuprate at 297 and 100 K. Acta. Cryst. C 1987, 43 (3), 413-416.

12. Yu, X. J.; Chi, X.; Wee, A. T. S.; Rusydi, A.; Breese, M. B. H. A Scripting Labview-Based Program for Experiment Automation in Synchrotron Radiation Applications. Rev. Sci. Instrum. 2019, 90 (10), 103902.

13. Breuer, T.; Klues, M.; Witte, G. Characterization of Orientational Order In П-Conjugated Molecular Thin Films by NEXAFS. J. Electron. Spectrosc. Relat. Phenom. 2015, 204, 102-115. 
14. Stöhr, J. NEXAFS Spectroscopy. Springer-Verlag Berlin Heidelberg GmbH: 1992.

15. Johs, B.; Herzinger, C. M.; Dinan, J. H.; Cornfeld, A.; Benson, J. D. Development of a Parametric Optical Constant Model for $\mathrm{Hg}_{1-\mathrm{X}} \mathrm{Cd}_{\mathrm{x}} \mathrm{Te}$ for Control of Composition by Spectroscopic Ellipsometry during MBE Growth. Thin Solid Films 1998, 313, 137-142.

16. Toll, J. S. Causality and the Dispersion Relation: Logical Foundations. Phys. Rev. 1956, $104(6), 1760$.

17. Asmara, T. C.; Wan, D.; Zhao, Y.; Majidi, M. A.; Nelson, C. T.; Scott, M. C.; Cai, Y.; Yan, B.; Schmidt, D.; Yang, M., et al. Tunable and Low-Loss Correlated Plasmons in Mott-like Insulating Oxides. Nat. Commun. 2017, 8, 15271.

18. Whitcher, T. J.; Zhu, J. X.; Chi, X.; Hu, H.; Zhao, D.; Asmara, T. C.; Yu, X.; Breese, M. B. H.; Castro Neto, A. H.; Lam, Y. M., et al. Importance of Electronic Correlations and Unusual Excitonic Effects in Formamidinium Lead Halide Perovskites. Phys. Rev. X 2018, 8 (2), 021034.

19. Maier, S. A. Plasmonics: Fundamentals and Applications. Springer US: 2007.

20. Yin, X.; Tang, C. S.; Zeng, S.; Asmara, T. C.; Yang, P.; Naradipa, M. A.; Trevisanutto, P. E.; Shirakawa, T.; Kim, B. H.; Yunoki, S. J. A. P. Quantum Correlated Plasmons and Their Tunability in Undoped and Doped Mott-Insulator Cuprates. ACS Photonics 2019, 6 (12), 32813289. 